\title{
BEAM DYNAMIC ASPECTS OF THE TESLA POWER COUPLER
}

\author{
M. Zhang and Ch. Tang* \\ CST GmbH, Lauteschlägerstraße 38, 64289 Darmstadt, Germany
}

\section{Abstract}

We studied the beam dynamic behavior of the RF power coupler of the TESLA superconducting cavities. It is found that the transverse RF kick is not negligible. A transverse beam wandering of 1 to $2 \mathrm{~mm}$ was predicted and subsequently observed on the TESLA Test Facility. Two proposals are presented to reduce the kicks.

\section{INTRODUCTION}

TESLA is a superconducting RF based large linear collider project [1]. Its 9 cell superconducting cavity operating at L-band is fed by a coaxial coupler [2]. Due to its nonsymmetrical layout, a transverse kick caused by electromagnetic fields is clearly inevitable. In order to address this issue, we conducted an intensive and systematic investigation into the complete dynamic process involving power feeding, bunch injection, kick accumulation, and emittance growth by means of both numerical and analytical methods.

The analytical and numerical results show a good agreement with each other, which are confirmed by the experiment. The transverse RF kick of the coupler is more than 120 times as strong as that of transverse wake fields of a TESLA 9 cell cavity by a $1 \mathrm{~mm}\left(\sigma_{z}\right) 1 \mathrm{nC}$ bunch at $1 \mathrm{~mm}$ off-axis. The time variation of the kicks is directly responsible for the emittance growth, which is as high as $27 \%$. The transverse offset at the exit of the first TESLA accelerating module in the TESLA Test Facility (TTF) is found to be $1 \sim 2 \mathrm{~mm}$ at $E_{0}=15 \mathrm{MeV}$ and $E_{a c c}=15 \mathrm{MV} / \mathrm{m}$.

With a view to reducing the transverse kicks, two proposals are presented. One is by adopting alternating coupler arrangement; The other by symmetrical coupler. Both RF kicks and emittance dilution can be reduced dramatically.

\section{SIMULATION APPROACH}

The simulation is carried with MAFIA [3] in two steps: T3 for field and TS3 for beam simulations.

\subsection{Field Simulation}

The geometry is shown in Fig. 1. RF power is fed at the outer terminal at $x=x_{\max }$. The two lowest cavity modes are E01-0 and E01- $\pi$.

\subsubsection{Field Ratio}

Table 1 lists $\hat{E}_{x}, c \hat{B}_{y}$, and $\hat{E}_{z}$ at different $Q_{l}$ values at 1 $\mathrm{W}$ input power. With this table, we derive the following

* Accelerator Laboratory, Department of Engineering Physics, Tsinghua University, Beijing, China, 100084

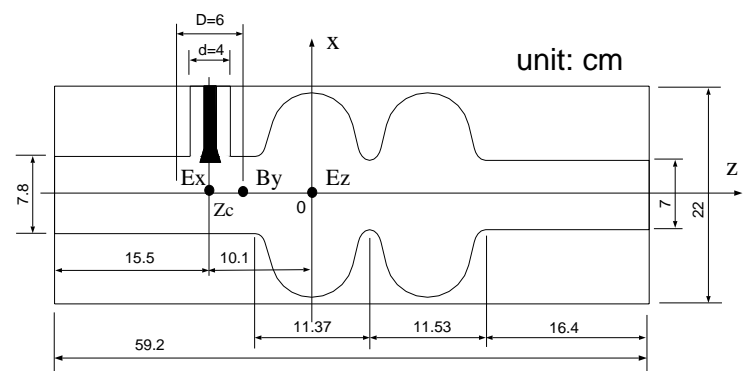

Figure 1: Geometry for time-domain simulations. At the three marked points, $E_{x}, B_{y}$, and $E_{z}$ are monitored.

Table 1: Amplitudes of $E_{x}, c B_{y}$, and $E_{z}$

\begin{tabular}{|c|c|c|r|}
\hline$Q_{l}$ & $\hat{E}_{x}(\mathrm{~V} / \mathrm{m})$ & $c \hat{B}_{y}(\mathrm{~V} / \mathrm{m})$ & $\hat{E}_{z}(\mathrm{~V} / \mathrm{m})$ \\
\hline 1000 & 110 & 13.5 & 400 \\
\hline 2000 & 110 & 15.0 & 800 \\
\hline 4000 & 110 & 16.5 & 1600 \\
\hline 6100 & 110 & 24.0 & 2700 \\
\hline
\end{tabular}

relations:

$$
\alpha_{E} \equiv \frac{\hat{E}_{x}}{\hat{E}_{z}} \approx \frac{10^{3}}{4 Q_{l}}, B \equiv \frac{c \hat{B}_{y}}{\hat{E}_{x}} \approx 0.075\left(2+\frac{Q_{l}}{10^{-4}}\right)
$$

With the above, we can obtain coupler fields at any $Q_{l}$ by extrapolation, thanks to the linear scaling law.

\subsubsection{Spatial and Temporal Dependence}

The spatial field distributions along the beam axis are shown in Fig. 2. As an approximation, we use following functions for the spatial dependence of $E_{x}$ and $B_{y}$ near the coupler,
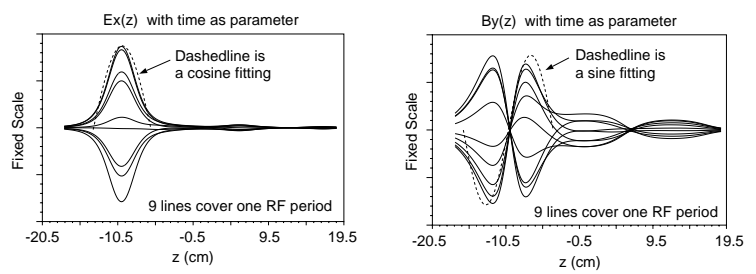

Figure 2: Spatial dependence of $E_{x}$ and $B_{y}$

$$
\begin{aligned}
& E_{x}(z)= \begin{cases}\hat{E}_{x} \cos \frac{\pi}{D}\left(z-z_{c}\right) & \left|z-z_{c}\right| \leq \frac{D}{2} \\
0 & \text { otherwise, }\end{cases} \\
& B_{y}(z)= \begin{cases}\hat{B}_{y} \sin \frac{\pi}{D}\left(z-z_{c}\right) & \left|z-z_{c}\right| \leq D \\
0 & \text { otherwise. }\end{cases}
\end{aligned}
$$


Figure 3 shows $E_{x}$ and $B_{y}$ phase distributions along axis at different $Q_{l}$ 's. For low Qs, $\phi_{E, l}=-\frac{\pi}{2}$ and $\phi_{B, l}=\pi$;
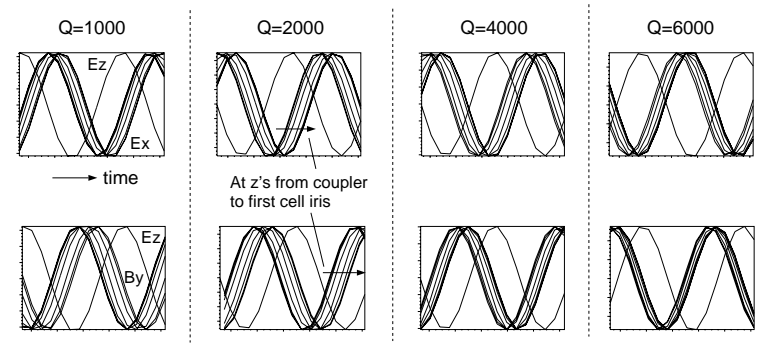

Figure 3: Temporal dependence of $E_{x}$ (top) and $B_{y}$ (bottom) with respect to $E_{z}$. Each plot has 8 curves which are monitored at 8 points along axis from $\mathrm{z}=-9.5$ to $-2.5 \mathrm{~cm}$.

While for high Qs, $\phi_{E, h}=-\pi$ and $\phi_{B, h}=\frac{\pi}{2}$.

A complete set of expressions for $E_{x}$ and $B_{y}$ near the coupler can be written as follows:

$$
\begin{gathered}
E_{x}(z, t)=\hat{E}_{x} \cos \frac{\pi}{D}\left(z-z_{c}\right) \cos \left(\omega t+\phi_{0}+\phi_{E}\right) \\
\left|z-z_{c}\right| \leq \frac{D}{2} \\
B_{y}(z, t)=\hat{B}_{y} \sin \frac{\pi}{D}\left(z-z_{c}\right) \cos \left(\omega t+\phi_{0}+\phi_{B}\right) \\
\left|z-z_{c}\right| \leq D
\end{gathered}
$$

where $\phi_{0}$ is injection phase. $\hat{E}_{x}$ and $\hat{B}_{y}$ are given by Eq. 1 .

\subsection{Kick Simulation}

A full 3D geometry is used for kick simulations. $Q_{l}$ is 6000. RF power of $1 \mathrm{MW}$ is fed at $\mathrm{t}=0$. A $1 \mathrm{nC} 5 \mathrm{MeV}$ bunch of length $20 \mathrm{ps}$ is injected at $\mathrm{t}=200 \mathrm{~ns}$ from either side of the cavity with $\phi_{0}=-45.6^{\circ}$ off-crest. The results are presented in Fig. 4. The beam divergences for both directions are

$$
x_{+z, \text { num }}^{\prime}=2.9 \times 10^{-4} \text { and } x_{-z, \text { num }}^{\prime}=5.8 \times 10^{-4} .
$$

\section{ANALYTICAL FORMULATION}

With the above derived equations, we obtain

$$
\begin{aligned}
P_{x}= & \frac{q \alpha_{E} \hat{E}_{z} d}{|c|} \frac{\frac{3}{\pi}}{1-\left(\frac{3 d}{\lambda}\right)^{2}} \\
& {\left[\cos \left(\pi \frac{3 d}{2 \lambda}\right) \cos \left(2 \pi \frac{z_{c}}{\lambda}+\phi_{0}+\phi_{E}\right)+\right.} \\
& \left.s \alpha_{B} \sin \left(\pi \frac{3 d}{\lambda}\right) \sin \left(2 \pi \frac{z_{c}}{\lambda}+\phi_{0}+\phi_{B}\right)\right] .
\end{aligned}
$$

For $-z$ beam direction, change $\lambda$ to $-\lambda, s$ from 1 to -1 , and $\phi_{0}$ to $\phi_{0}+\pi$.
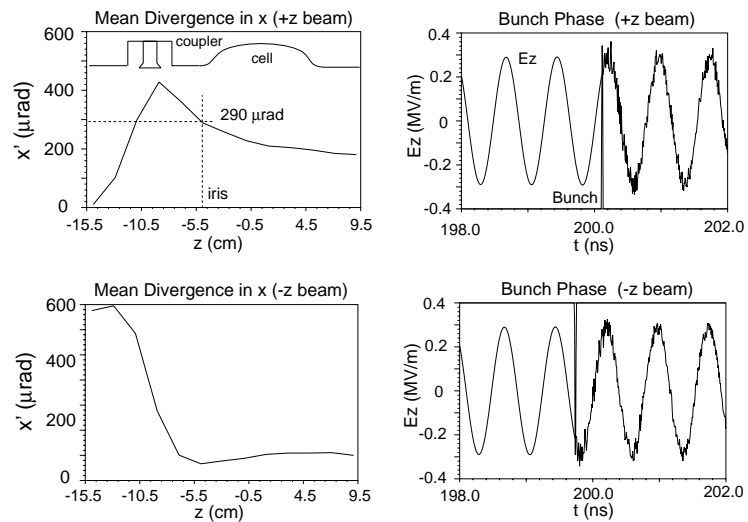

Figure 4: Kick simulation results. Top row: $+\mathrm{z}$ beam direction; Bottom: - $\mathrm{z}$ beam.

By inserting the parameters used in Section 2.2 into Eq. 7, $\hat{E}_{x}=1.12 \times 10^{5}, \alpha_{B}=\frac{c \hat{B}_{y}}{\hat{E}_{x}}=0.112$, we get

$$
x_{+z, a n a}^{\prime}=2.6 \times 10^{-4} \text { and } x_{-z, a n a}^{\prime}=6.1 \times 10^{-4} .
$$

Comparing with the numerical results (Eq. 6), we find they agree within $10 \%$. This nice agreement provides a solid basis for an extrapolation to high Q cases.

The RF kick for an actual TESLA cavity is estimated as follows. Given $Q_{l}=5 \times 10^{6}, \hat{E}_{z}=30 \mathrm{MV} / \mathrm{m}, \phi_{0}=$ $-15^{\circ}, d=4 \mathrm{~cm}, \lambda=23 \mathrm{~cm}, z_{c}=-10.1 \mathrm{~cm}$, we get (Beam goes in $-\mathrm{z}$ )

$$
\begin{aligned}
P_{x} & =2.9 \times \cos \left(2 \pi \frac{z_{c}}{\lambda}+\phi_{0}\right) \quad(\mathrm{keV} / \mathrm{c}) \\
& =2.9 \times \cos \left(2 \pi \frac{-10.1}{-23}+\pi+\phi_{0}\right)=2.3 \quad(\mathrm{keV} / \mathrm{c}) .
\end{aligned}
$$

This value is more than 120 times stronger than transverse kick factor $k_{t}^{9 c e l l}$, which is $18 \mathrm{~V} / \mathrm{nC} / \mathrm{mm}$ for a $1 \mathrm{~mm}\left(\sigma_{z}\right)$ $1 \mathrm{nC}$ bunch [4]. Another equivalence can be made to the wake field kick $\left(k_{t}^{c p l r}\right)$ of the coupler itself. Simulation shows $k_{t}^{c p l r}=\frac{k_{t}^{9 c e l l}}{5.3}$ for a $1 \mathrm{~mm} \sigma_{z}$ bunch, i.e. $k_{t}^{c p l r}=3.4$ $\mathrm{V} / \mathrm{nC}$ (on-axis). To reach the $2.3 \mathrm{keV} / \mathrm{c} \mathrm{RF}$ kick, the bunch charge should be as high as $676 \mathrm{nC}$ !

Relative emittance growth can be estimated with

$$
\frac{d \epsilon_{n, x}}{\epsilon_{n, x}}=\left(1+\alpha^{2}\right)\left(\frac{d \sigma_{x}}{\sigma_{x}}+\frac{d \sigma_{P_{x}}}{\sigma_{P_{x}}}-\frac{d \sigma_{x P_{x}}}{\sigma_{x P_{x}}}\right)+\frac{d \sigma_{x P_{x}}}{\sigma_{x P_{x}}} .
$$

Assume that the coupler kick is instantaneous, then $d \sigma_{x}=0$. If the bunch comes into the coupler fields with $\alpha=0$, Eq. 10 can be simplified to

$$
\frac{d \epsilon_{n, x}}{\epsilon_{n, x}}=\frac{d \sigma_{P_{x}}}{\sigma_{P_{x}}}, \text { with } d \sigma_{P_{x}}=\frac{d P_{x}}{d \phi_{0}} \sigma_{z},
$$

where $\sigma_{z}$ is rms bunch length. With Eq. 9, we get $\frac{d P_{x}}{d \phi_{0}}=$ $1.74(\mathrm{keV} / \mathrm{c})$. At the entrance of the first cryomodule, $\sigma_{z}=1 \mathrm{~mm}$, so $d \sigma_{P_{x}}=47 \mathrm{eV} / \mathrm{c}$, i.e. $d \sigma_{\left(\gamma \beta_{x}\right)}=9.2 \times 10^{-5}$. Assume $\epsilon_{n, x}=0.6 \pi \mathrm{mm}-\mathrm{mrad}$ and $\sigma_{x}=0.2 \mathrm{~mm}$ (TTF 


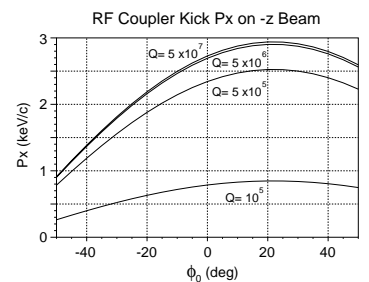

Figure 5: $P_{x}\left(\phi_{0}, Q_{l}\right)$. At certain $Q_{l}, P_{x}$ may vanish.

FEL $0.5 \mathrm{nC}$ optimized case [5]), it yields $\sigma_{\left(\gamma \beta_{x}\right)}=3 \times$ $10^{-3}$. And finally, we have

$$
\frac{d \epsilon_{n, x}}{\epsilon_{n, x}}=\frac{d \sigma_{P_{x}}}{\sigma_{P_{x}}}=\frac{d \sigma_{\left(\gamma \beta_{x}\right)}}{\sigma_{\left(\gamma \beta_{x}\right)}}=\frac{9.2 \times 10^{-5}}{3 \times 10^{-3}} \approx 3 \% .
$$

For the cryomodule of eight couplers, there will be as high as $27 \%$ emittance growth. For FEL operation, this number is obviously not small. $P_{x}$ shows a strong dependence on $\phi_{0}$ and $Q_{l}$ (Fig. 5).

\section{ESTIMATION OF RF DISPLACEMENT IN THE FIRST TTF CRYOMODULE}

The TESLA accelerating module (cryomodule, Fig. 6) is composed of eight cavities, each of which has an RF power coupler. The couplers are mounted at the downstream end of the cavities. We traced a single particle through the
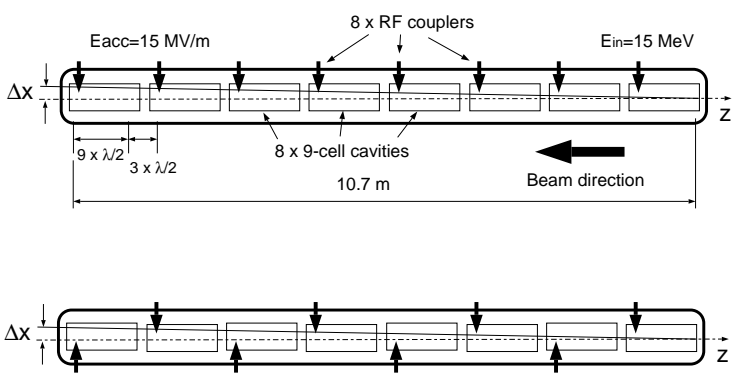

Figure 6: Top: Original TESLA cryomodule; Bottom: Alternating RF coupler placement - A proposal that reduces beam displacement by a factor of 5.3.

structure with lumped transverse kicks from the couplers. The accumulated offset $\Delta x$ is $1 \sim 2 \mathrm{~mm}$. It has a strong dependence on $\phi_{0}$ and $Q_{l}$. Since the absolute value of RF kicks is independent of initial beam energy, it is therefore more harmful at low beam energies. The displacement was confirmed by measurement [6].

\section{SYMMETRICAL RF POWER COUPLER AND SUPER $^{2}$ STRUCTURE}

The RF kick comes from non-symmetrical stationary field pattern near coupler. A natural solution is to use a symmetrical coupler. With particle simulations, emittance growth can be reduced by $17 \%$. We propose to use such symmetrical coupler to feed two superstructures [7] in the middle,

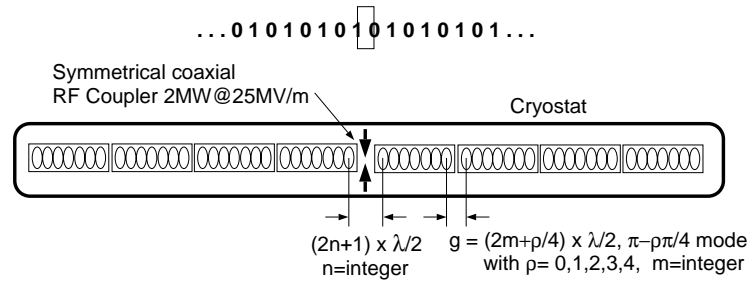

Figure 7: Super $^{2}$ structure proposal for TESLA. The symmetrical coupler ought to provide a $2 \mathrm{MW}$ RF power, 1 MW for 4 cavities at $10 \mathrm{~mA}$ beam current [8]. Mode "a-b" is defined as (cell-to-cell)-(cavity-to-cavity) phase advance.

so called super ${ }^{2}$ structure (Fig. 7). Effective accelerating length of a super ${ }^{2}$ structure is $13 \%$ higher than that of the original structure. Given $n=1, \rho=0$, and $m=1$, the net geometrical length of a super ${ }^{2}$ structure is $8 \times 8=64$ cells; Accelerating length is $8 \times 7=56$ cells. For the original structure, they are $8 \times 12-3=93$ cells and $8 \times 9=72$ cells, respectively. The gain ratio is then

$$
\frac{\text { super }^{2} \text { structure }}{\frac{56}{64}}=1.13 \text {. }
$$

\section{CONCLUSIONS}

We made a systematic investigation of transverse kicks on beams by the TESLA RF power coupler. The analytical and numerical results agree quite well with each other. The theoretical prediction was confirmed by the experiment. The accumulated beam displacement in the TTF cryomodule is $1 \sim 2 \mathrm{~mm}$. Emittance growth is found to be as high as $27 \%$. The proposed super ${ }^{2}$ structure can not only exploit the effective accelerating length of TESLA, but also dramatically reduce the RF kicks.

\section{REFERENCES}

[1] R. Brinkmann, G. Materlik, J. Rossbach, A. Wagner (eds.), Conceptual Design of a $500 \mathrm{GeV}$ e+e- Linear Collider with Integrated X-ray Laser Facility, DESY 1997-048 and ECFA 1997-182

[2] D. Proch, P. Schmüser (eds.), TESLA Input Coupler Workshop, DESY Print, TESLA Report 96-09, August 1996

[3] MAFIA version 4.013, CST GmbH, Lauteschlägerstraße 38, D-64289 Darmstadt, Germany, http://www.cst.de

[4] A. Mosnier, Longitudinal and Transverse Wakes for the TESLA Cavity, DESY Print, TESLA Report 93-11, May 1993

[5] M. Zhang, T. Limberg, PARMELA and COMFORT optimization results

[6] S. Fartoukh and et al, RF Kick Measurement on TTF and Comparison with the TESLA Specifications, CEA/DAPNIA/SEA-98-42

[7] J. Sekutowicz, M. Ferrario, Ch. Tang, Superconducting Superstructure for TESLA Collider, DESY Print, TESLA Report 98-08, April 1998

[8] D. Proch, TESLA collaboration meeting, March 1998 